J. Clin. Chem. Clin. Biochem.

Vol. 15, 1977, pp. 339-343

\title{
Vereinfachte titrimetrische Halbmikrobestimmung der Lipase im Serum
}

\author{
Von W. Appel und H.-G. Scholz \\ Zentrallaboratorium und Medizinische Klinik (Leiter: Prof. Dr. med. P. M. Reisert) der St.-Vincentius-Krankenhäuser \\ Karlsruhe
}

(Eingegangen am 14. April 1976/11. Februar 1977)

Zusammenfassung: Die quantitative, titrimetrische, kinetische Bestimmungsmethode für die Lipase in Serum, Duodenalsaft und Punktaten nach Rick (Rick, $W$. (1969), diese Z. 7, 530-539) wird als Halbmikromethode mit 0,20 $\dot{m} \mathrm{l}$ Probenvolumen ohne $\mathrm{N}_{2}$-Schutzgas für Routinezwecke adaptiert. Methodenbeschreibung und Qualitätssicherungsdaten werden mitgeteilt. Die Methode hat sich im Verlauf von mehr als 3 Jahren bei über 3000 Patienten bewährt.

\section{Simplified titrimetric semimicrodetermination of lipase in serum}

Summary: The quantitative, titrimetric and kinetic method for the determination of lipase in serum, duodenal juice and punctures by Rick (Rick, W. (1969), this journal 7, 530-539) has been adapted as a routine semimicro-method using $0.20 \mathrm{ml}$ volume of specimen without $\mathrm{N}_{2}$ protection. The description of the procedure and quality control data are reported. The method has been checked for more than 3 years and sera from more than 3000 patients have been investigated.

\section{Einführung}

„Längsprofile“ (Langzeitbeobachtungen) der Lipase über Monate hinweg sind bislang kaum bekannt geworden. Ihre Kenntnis beim einzelnen Patienten erlaubt eine Beurteilung der bekannten definierten Pankreaserkrankungen, eine empfindliche, präzise und richtige Bestimmungsmethode im ,Normal“- und „Ubergangsbereich" vorausgesetzt. Dies gilt gleichermaßen für die Erfassung atypischer Krankheitsbilder wie auch für die Kontrolle akut und chronisch reżidivierender Verlaufsformen sowie für die postoperative Ubberwachung bei Eingriffen im Oberbauch. Diese Beurteilung mittels eines Längsprofiles erscheint uns zur Klärung der Verlaufsform und zư Uberprüfung der eingeschlagenen Therapie besonders im Hinblick auf das wenig spezifische und flüchtige Verhalten der $a$-Amylase-Aktivität erforderlich.

Als Voraussetzung betrachteten wir: Eine Vereinfachung der Bestimmungsmethode nach Rick (1) zur routinemäßigen Praktikabilität unter Erhạlt der Spezifität; die umfassende methodologische Prüfung der Modifikation; eine hinreichende lange Erprobungszeit und eine kritische Korrelation mit der Klinik in einer genügend großen Zahl unausgewählter Einzelfälle. Entscheidend wäre allerdings der Vergleich mit einer Referenzmethode, die jedoch noch nicht vorliegt.

Die methodischen Einzelheiten werden nachfolgend, klinische Ergebnisse an anderer Stelle beschrieben.

\section{Methodenbeschreibung ${ }^{1}$ )}

Prinzip der Methode und Ablauf der Reaktion: 1. c. (1)

Geräte

Titrierausrüstung „Radiometer" bestehend aus: Titrator TTT 2b, Registrierschreiber SBR 3, Autobürette ABU 12, Halbmikro-Titriereinheit TTA 31, Titriergefäße $V 525$ aus Plastik mit Magnetrührplättchen, Füllvolumen $0,5-4,0 \mathrm{ml}$, Thermostatiergefäß V 526, Halbmikroglaselektrode G 2222 C und Kalomelelektrode K 4112, offenes Wasserbad mit Leitungswasserkühlung; Reaktionsgefäße (Titrierbecher) aus Glas oder Plastik.

Für Spezialuntersuchungen: Stickstoff-Gasflasche mit Druckmischer, Gaswaschflasche mit $\mathrm{H}_{2} \mathrm{SO}_{4}$, Natronkalkturm, Filter und Blasenzähler (2 Blasen/s); Starmix o. ä., Glaspipetten mit ausgezogener Spitze, 0,200, 0,500 und 1,00 ml; Vollpipetten $2,000 \mathrm{ml}$; Mikropipetten $20 \mathrm{\mu l}$.

1) Nach den Vorlagen der Standardisierungskommission der Deutschen Gesellschaft für Klinische Chemie (März 1975) sowie DIN-Entwurf 58937 Teil 4 (Januar 1975). 


\section{Reagenzien}

Gummi arabisch, ausgelesen, fein gepulvert, z. B. Merck, Art. 4282 , oder entsprechende Qualität; Olivenöl DAB 7, (besser DAB 6), z. B. Olivenöl DAB 7/ÖAB 9/BP, Firma Henry Lamotte, Bremen; Glykocholsäure, Na-Salz; Bidestilliertes Wasser, frisch, $\mathrm{CO}_{2}$-frei; $\mathrm{NaOH}$-Lösung, $1,0,0,1$ und $0,01 \mathrm{~mol} / \mathrm{l} ; \mathrm{HCl}$ Lösung, $1,0 \mathrm{~mol} / \mathrm{l}$; Standard-Pufferlösung, z. B. Radiometer $\mathrm{S} 1510, \mathrm{pH} 7,383$ bei $25^{\circ} \mathrm{C}$

\section{Herstellung der Lösungen}

Lösung 1: Gummi-arabicum-Lösung (Stammlösung ${ }^{2}$ )). $50 \mathrm{~g}$ Gummi arabicum und $450 \mathrm{ml}$ bidest. Wasser im Starmix (Stufe I) so lange mischen, bis die Substanz vollständig gelöst ist (etwa $15 \mathrm{~min}$ ); Lösung in einen $500 \mathrm{ml}$ Meßzylinder überführen, mit bidest. Wasser auf $500 \mathrm{ml}$ auffüllen und gut mischen. Nach einigen Stunden die über dem Bodensatz befindliche, schwach getrübte Lösung in eine Polyethylenflasche abgießen und bei $+4^{\circ} \mathrm{C}$ aufbewahren.

Lösung 2: Olivenölemulsion, etwa $200 \mathrm{~g} / \mathrm{l}$.

$400 \mathrm{ml}$ Lösung $1 \mathrm{mit} 100 \mathrm{ml}$ Olivenöl mischen, etwa $5,0 \mathrm{ml}$ $1 \mathrm{~mol} / \mathrm{l} \mathrm{NaOH}$ zugeben und nochmals $2 \mathrm{~min}$ homogenisieren.

In die Substratemulsion anschließend etwa 1,0-1,5 ml $1 \mathrm{~mol} / 1 \mathrm{HCl}$ tropfenweise zugeben, $1 \mathrm{~min}$ homogenisieren; der $\mathrm{pH}$-Wert soll etwa 8,2 betragen. Die Emulsion in etwa 25 ml-Portionen abfüllen und gut verschlossen aufbewahren.

Lösung 3: Na-Glykocholatlösung, $75 \mathrm{mmol} / \mathrm{l}$.

$366 \mathrm{mg}$ Glykocholsäure, Na-Salz, in $10,0 \mathrm{ml}$ bidest. Wasser lösen, Lösung in 10-15 Portionen abfüllen und bei $+4^{\circ} \mathrm{C}$ aufbewahren.

Lösung 4: Bidest. Wasser, Kohlendioxid-frei.

Bidest. Wasser in einer Glasschliff-Kochflasche mehrere Minuten bei $100^{\circ} \mathrm{C}$ halten, mit Natronkalkrohr verschließen und abkühlen lassen.

Lösung 5: (Titrant): $\mathrm{NaOH}-L o ̈ s u n g, ~ 0,01 \mathrm{~mol} / \mathrm{l}$.

In einen $100 \mathrm{ml}-\mathrm{Meßkolben}$ bis zur Marke $0,1 \mathrm{~mol} / 1 \mathrm{NaOH}$ Lösung einfüllen, diese quantitativ in einen $1000 \mathrm{ml}-\mathrm{Meß}$ kolben überführen und bis zur Marke mit Lösung 4 auffüllen; gut mischen und in einer Vorratsflasche mit Natronkalkverschluß aufbewahren.

Haltbarkeit der Lösungen $\left(4^{\circ} \mathrm{C}\right)$

Lösung 1 und 5: mindestens 2 Monate

Lösung 2: $\quad$ maximal 3 Tage

Lösung 3: $\quad$ maximal 3 Monate

Standard- und Kontrollproben

Interne Standards für Enzyme sind bislang noch nicht definiert. Als Kontrollen dienen im Handel befindliche Kontrollseren, vor allem Validate $\mathbf{N}$ und - A (Gödecke AG, Berlin-Freiburg), oder Enzatrol (Merz + Dade GmbH, München).

\section{Vorbereitung des Patienten}

Keine besonderen Maßnahmen erforderlich, im Akutfall ohnehin nicht möglich. Heparinpräparate, Opiate und Corticoide stellen mögliche Störfaktoren dar. Bei Durchführung von Provokationsoder Evokationstesten sind die hierbei erforderlichen Maßnahmen zu beachten.

\section{Vorbereitung der Geräte}

Die Vorwärmzeit der Geräte beträgt etwa $5 \mathrm{~min}$, die des Wasserbades je nach Kapazität etwa 5-15 min; Justierungsmaßnahmen sind nicht erforderlich.

\section{Vorbereitung der Reagenzien}

Alle Lösungen müssen auf $25^{\circ} \mathrm{C}$ vorge wärmt sein oder werden; die Titerkorrektur sollte wöchentlich erfolgen. Die Verwendung einer Checkliste (siehe Tab. 1) wird empfohlen.

2) Die Firma Merck stellte uns freundlicherweise eine Stammlösung mit $160 \mathrm{~g} / 1$ zur Verfügung, wofür an dieser Stelle ausdrücklich gedankt sei.
Tab. 1. Checkliste.

1. Apparateaufstellung (Verbindung zivischen Titrator und Bürette; Elektrodenkabel) einwandfrei?

2. Geräte und Wasserbad angewärmt?

3. Temperatur kontrolliert: $25,0 \pm 0,1^{\circ} \mathrm{C}$ ? Thermometer geprüft?

4. Bei $\mathrm{pH} \doteq 7,383$ „Buffer adjustment" etwa in Mittelstellung?

5. "Endpoint" 8,20 eingestellt?

6. Apparateeinstellung mit ,Stand-by“ kontrolliert? (Tab. 2)

7. Titer in Ordnung? (wöchentliche Kontrolle)

8. Tintenschreiber eingesetżt und funktionsfähig?

9. Kolben der Bürette mit $\mathrm{NaOH}$ gespült (,,empty burette") und gefullt?

10. Titrant-Zufuhrsystem luftbläsenfrei?

11. Titrationseinheit sauber, trocken und dicht?

12. Planschliff des Dreiwegehahnes der Kolbenbürette sauber und dicht?

13. Rührvorrichtung (Magnete, Treibriemen und Übersetzungsverhältnis) kontrolliert?

14. Elektroden fettfrei, einwandfrei ansprechend und richtig eingesetzt?

15. Vorwärmung von Proben und Kontrollprọben ausreichend?

Entnahme des Untersuchungsgutes und der Untersuchungsprobe

Venenblut ohne Z̄usätże; für Duodenalsaft, Ascitespunktate u. a. gelten die Vorschriften bei der Durchfuhrung von Provokationstesten (z. B. Kühlung).

Auffang- und Versandgefäße, Verwahrung, Transport des Untersuchungsgutes

Keine besonderen Vorschriften; empfohlen werden die auch für die üblichen klinisch-chemischen Untersuchungen geeigneten Probenröhrchen mit Trennmittel, z. B. Sarstedt 95/24 L. Zugabe von gerinnungsfördernden Mitteln (Kaolin) ist möglich, von gerinnungshemmenden Mitteln unnötig. Für Duodenalsaft u.a. gelten Sondervorschriften (z. B. Kühlung).

Aufbereitung des Untersuchungsgutes

Die Serumgewinnung erfolgt wie üblich; anderes Untersuchungsmaterial muß klar zentrifugiert werden. Temperaturen unter $35^{\circ} \mathrm{C}$ und Licht besitzen keinën Einfluß. Duodenalsaft muß sofort gekühlt $\left(0^{\circ} \mathrm{C}\right)$ werden; die Messung hat innerhalb $3 \mathrm{~h} \mathrm{zu}$ erfolgen (2).

Verwahrung der Proben

Die Proben können bèi $-20^{\circ} \mathrm{C}$ monatelang, bei $4^{\circ} \mathrm{C}$ mindestens zwei Wochen, bei Raumtemperatur mindestens $17 \mathrm{~h}$ aufbewahrt werden; Luft und Licht besitżen keinen Einfluß; Zusatz von Konservierungsmitteln ist nicht erforderlich. Dies gilt nicht für Duodenalsaft und andere Körperflüssigkeiten, die wegen der Autolysegefahr möglichst frisch verarbeitet werden müssen.

\section{Arbeitsweise}

Checkliste (Tab; 1) beachten.

In einem Titrierbecher Rührmagnet mit Zacken nach oben einlegen und pipettieren:

\begin{tabular}{lccc}
\hline Lösungen & $\begin{array}{l}\text { Reagenzien- } \\
\text { leerwert }\end{array}$ & Probe & \multicolumn{1}{l}{$\begin{array}{l}\text { Kontroll- } \\
\text { probe }\end{array}$} \\
\hline Lösung 2 & $20 \mu \mathrm{l}$ & $20 \mu \mathrm{l}$ & $20 \mu \mathrm{l}$ \\
Lösung 3 & $2000 \mu \mathrm{l}$ & $2000 \mu \mathrm{l}$ & $2000 \mu \mathrm{l}$ \\
Lösung 4 & $200 \mu \mathrm{l}$ & - & - \\
Probe & - & $200 \mu \mathrm{l}$ & $200 \mu \mathrm{l}$ \\
\hline
\end{tabular}

sofort durch rasche Zugabe (200-400 $\mu \mathrm{l} / \mathrm{min})$ von Lösung 5 auf $\mathrm{pH} 8,2$ titrieren und langsamer $(1,25-50,0 \mu \mathrm{l} / \mathrm{min})$. 2-5 min, mindestens bis zum Erreichen einer linearen Kinetik weitertitrieren. Patientendaten auf Registrierpapier vermerken. 
Bei Titrationsdauer über 10 min Ansatz mit $500 \mu l$ Probe wiederholen.

In der $Z$ wischenzeit ein weiteres Reaktionsgefäß vorbereiten: Reinigen der Titrierbecher mit Wasser, Aceton und Benzin (Wattebausch); nach mehrminütigem Lufttrocknen beschicken.

Berechnung

Katalytische Konzentration

$[\mathrm{U} / 1]=\left(\mathrm{T}_{\mathbf{x}}[\mu \mathrm{l} / \mathrm{min}]-\mathrm{T}_{\mathrm{o}}[\mu \mathrm{l} / \mathrm{min}]\right) \mathrm{F} \cdot \mathrm{V}$

Für $T_{\mathbf{x}}=$ Natronlaugeverbrauch des Probeansatzes aus dem linearen Kurvenanstieg auf dem Registrierpapier, in $\mu \mathrm{l} / \mathrm{min}$;

$\mathrm{T}_{\mathrm{o}}=$ ebenso, aus dem Reagenzienleerwertansatz;

$\mathrm{F}=$ Produkt aus Umrechenfaktor und Titer der Lösung 5, meist 47-53;

V = Verdünnungsfaktor der Probe, falls erforderlich.

\section{Zuverlässigkeitskriterien}

Präzision: Die Ergebnisse sind in Tabelle 3 zusammengestellt. Der Variationskoeffizient (VK) von Tag zu Tag betrug bei Validate $\mathrm{N}$ an 260 Tagen $18,8-28,5 \%$, bei Enzatrol an 291 Tagen 12,1-19,5\%.

Richtigkeit: Linearität (Zeit, Menge): Die Reaktion verläuft 0,5-3 min nach dem Start über wenigstens $2 \mathrm{~h}$ nach der Kinetik einer Reaktion 0-ter Ordnung. Im Bereich von 5-800 U/1 besteht lineare Beziehung $\mathrm{zwischen}$ Meßsignal und katalytischer Konzentration von Lipase; dies gilt entsprechend auch für 1:10-, 1:100- und 1:1000-Verdünnungen (Duodenalflüssigkeit). Bei zwei Patienten mit akuter Pankreatitis und Lipasewerten um $3000 \mathrm{U} / 1 \mathrm{nahm}$ die spezifische Aktivität beim Verdünnen zu. Die Ursache ist unbekannt.

Wiederfindungsversuch: nicht durchgeführt.

Spezifität: siehe 1. c. (1). Auf die Problematik der Colipasen I und II (23) kann hier nicht eingegangen werden.
Tab. 2. Apparateeinstellung.

\begin{tabular}{|c|c|}
\hline \multicolumn{2}{|l|}{ pH-Meter TTT 2} \\
\hline $\begin{array}{l}\text { Puffer-Range: } \\
\text { Delay: } \\
\text { Proportionalband: } \\
\text { Endpoint: } \\
\text { Skala: } \\
\text { Temperatur: }\end{array}$ & $\begin{array}{l}0-14 \\
\text { unendlich } \\
0,05 \mathrm{pH} \\
8,60 \\
\operatorname{up}_{25^{\circ} \mathrm{C}}\end{array}$ \\
\hline \multicolumn{2}{|l|}{ pH-Modul PHA 993} \\
\hline $\begin{array}{l}\text { pH: } \\
\text { Int. Chart Calibrat.: }\end{array}$ & $\begin{array}{l}\text { pH-Stat } \\
0,1 \mathrm{pH}\end{array}$ \\
\hline \multicolumn{2}{|l|}{ Autobürette $A B U 12$} \\
\hline $\begin{array}{l}\text { Titrant: } \\
\text { Increments: } \\
\text { Speed: }\end{array}$ & $\begin{array}{l}0,1 \mathrm{n} \mathrm{NaOH}, \text { faktorkorrigiert } \\
\text { off } \\
40\end{array}$ \\
\hline \multicolumn{2}{|c|}{ Registrierschreiber SBR 3} \\
\hline $\begin{array}{l}\text { Pen movement: } \\
\text { Chart feed: }\end{array}$ & $\begin{array}{l}x 1 \\
1 \mathrm{~cm} / \mathrm{min}\end{array}$ \\
\hline
\end{tabular}

Meßbereich: die Nachweisgrenze (aus $\mathrm{T}_{\mathrm{o}}$ in Serie) liegt je nach Leerwert und Reaktionszeit - um $5 \mathrm{U} / 1$; bei katalytischen Konzentrationen über $800 \mathrm{U} / 1$ sollen Verdünnungen mit $\mathrm{NaCl}$-Lösungen, $9 \mathrm{~g} / \mathrm{l}$, angesetzt werden. Der Leerwert hängt bei intaktem System nur von der Qualität der Substratemulsion ab, vor allem von ihrem Alter und von der Reinheit von Gummi arabicum und Olivenöl.

Als praktische Nachweisgrenze verwenden wir $10 \mathrm{U} / 1$. Methodenbeeinflussung: Hämolyse stört entgegen 1.c. (3) nicht. Folgende Pharmaka und körpereigene Substanzen zeigen keinen Einfluß (Endkonzentrationen im Serum nach $5 \mathrm{~min}$ Vorinkubation bei $25^{\circ} \mathrm{C}$ ): Bilirubin $(100 \mathrm{mg} / \mathrm{l})$, Glucose $(8 \mathrm{~g} / \mathrm{l})$, Fructose $(2 \mathrm{~g} / \mathrm{l})$, Acetazolamid (Diamox. $25 \mathrm{~g} / 1$ ), Gentamicin (Refobacin, $20 \mathrm{~g} / 1$ ),

Tab. 3. Qualitätssicherungsdaten: Angabe der Differenz zwischen deklariertem Wert $m_{o}$ und gefundenem Mittelwert $\bar{x}$ zu $\left.\left[\mathrm{m}_{\mathbf{0}}-\overline{\mathbf{x}}\right): \mathrm{m}_{\mathbf{0}}\right] \times 100$.

\begin{tabular}{|c|c|c|c|c|c|c|c|c|c|c|c|c|}
\hline \multirow{2}{*}{$\begin{array}{l}\text { Sta- } \\
\text { tistische } \\
\text { Kenn- } \\
\text { größe }\end{array}$} & \multicolumn{6}{|c|}{ Validate N Charge 2991103} & \multicolumn{4}{|c|}{ Enzatrol, Charge ET-243 } & \multirow{2}{*}{$\begin{array}{l}\text { Patho- } \\
\text { trol }{ }^{2} \text { ) } \\
1-6 / 76\end{array}$} & \multirow{2}{*}{$\begin{array}{l}\left.\text { Pool }^{3}\right) \\
1975\end{array}$} \\
\hline & $\begin{array}{l}\text { in } \\
\text { Serie }\end{array}$ & $1-6 / 74$ & $6-12 / 74$ & $1-6 / \overline{7} 5$ & $6-11 / 75$ & $\left.3 / 76^{1}\right)$ & $\begin{array}{l}\text { in } \\
\text { Serie }\end{array}$ & 1974 & $1-6 / 75$ & $-3 / 76$ & & \\
\hline $\begin{array}{l}\overline{\bar{x}}[\mathrm{U} / 1] \\
\mathrm{s}[\mathrm{U} / \mathrm{l}] \\
\mathrm{VK}[\%] \\
\mathrm{n} \\
\text { Verteilung } \\
\text { Diffe- } \\
\text { renz [\%] }\end{array}$ & $\begin{array}{c}68,2 \\
3,24 \\
4,75 \\
20 \\
20 \\
+\quad 6,56\end{array}$ & $\begin{array}{c}73,0 \\
19,0 \\
26,0 \\
59 \\
\text { norm } \\
+14,1\end{array}$ & $\begin{array}{c}66,5 \\
15,7 \\
24,5 \\
51 \\
\text { norm } \\
+\quad 9,0\end{array}$ & $\begin{array}{c}70,4 \\
13,1 \\
1.8,8 \\
106 \\
\text { normín } \\
+10,0\end{array}$ & $\begin{array}{c}53,1 \\
23,6 \\
44,4 \\
64 \\
\text { noimm } \\
-17,0\end{array}$ & $\begin{array}{c}65,6 \\
18,7 \\
28,5 \\
44 \\
- \\
-13,4\end{array}$ & $\begin{array}{l}575 \\
55,0 \\
9,56 \\
25 \\
= \\
-\end{array}$ & $\begin{array}{l}507 \\
61,3 \\
12,1 \\
40 \\
\text { norimi } \\
-\end{array}$ & $\begin{array}{l}500 \\
86,0 \\
17,2 \\
105 \\
\text { norm } \\
-\end{array}$ & $\begin{array}{c}549,0 \\
106,9 \\
19,5 \\
146 \\
\text { norm } \\
-\end{array}$ & $\begin{array}{l}112 \\
33,1 \\
30,0 \\
12 \\
- \\
-\end{array}$ & $\begin{array}{l}60,2 \\
11,9 \\
19,8 \\
91 \\
- \\
-\end{array}$ \\
\hline
\end{tabular}

Präzision: Validate $(n=260): \mathrm{VK}=18,8-28,5 \%$

Enzatrol $(n=291): V K=12,1-19,5 \%$

1) Charge 560064; bei $4{ }^{\circ} \mathrm{C}$ nach 2 Jahren nicht mehr stabil

2) Charge PT $69 \mathrm{~A}$, bei $4^{\circ} \mathrm{C}$ nicht stabil gegen Ende der Untersuchungsperiode

3) Gepooltes Serum von Patienten ohne Anhaltspunkt auf Pankreaserkrankungen, abgefüllt zu 1,5 ml-Proben und aufbewahrt bei $-20^{\circ} \mathrm{C}$. Jede Probe nur einmal verwendet. 
Tab. 4. Normbereiche für die katalytische Konzentration von Lipase im Serum.

\begin{tabular}{|c|c|c|c|c|c|c|}
\hline Kollektiv & $\mathrm{n}$ & $\begin{array}{l}\bar{x} \\
{[U / 1]}\end{array}$ & $\begin{array}{l}\mathrm{s} \\
{[\mathrm{U} / \mathrm{I}]}\end{array}$ & $\begin{array}{l}\text { Bereich } \\
-2 \mathrm{~s} \leqq \overline{\mathrm{x}} \\
\leqq+2 \mathrm{~s} \\
{[\mathrm{U} / 1]}\end{array}$ & $\begin{array}{l}\text { Ver- } \\
\text { teilung }\end{array}$ & Autor \\
\hline $\begin{array}{l}\text { Pankreas- } \\
\text { gesunde }\end{array}$ & 126 & 51 & - & $18-141$ & lognorm & (1) \\
\hline $\begin{array}{l}\text { Stat. } \\
\text { Patienten }{ }^{1} \text { ) }\end{array}$ & 381 & 60 & - & $23-162$ & lognorm & (1) \\
\hline $\begin{array}{l}\text { Blut- } \\
\text { spender }^{2} \text { ) }\end{array}$ & 68 & 78 & 29 & $20-136$ & - & (7) \\
\hline $\begin{array}{l}\text { Blut- } \\
\text { spender }^{3} \text { ) }\end{array}$ & 24 & 115 & 40 & $35-195$ & - & (7) \\
\hline $\begin{array}{l}\text { Kinder, } \\
\text { 0-2 Jahre }{ }^{2} \text { ) }\end{array}$ & 20 & 57 & 24 & $9-105$ & - & (7) \\
\hline Blutspender & 100 & 64,5 & - & $23-183$ & - & (8) \\
\hline $\begin{array}{l}\text { Pankreas- } \\
\text { gesunde }\end{array}$ & 57 & - & - & (bis 165) & - & (9) \\
\hline $\begin{array}{l}\text { Pankreas- } \\
\text { gesunde }^{3} \text { ) }\end{array}$ & 184 & - & - & (bis 182) & - & (10) \\
\hline $\begin{array}{l}\text { Stat. Pa- } \\
\text { tienten }^{1} \text { ) }\end{array}$ & 68 & - & - & (ca. 150) & - & (11) \\
\hline $\begin{array}{l}\text { Stat. Pa- } \\
\text { tienten }^{1} \text { ) }\end{array}$ & 141 & - & - & (bis 165) & - & (12) \\
\hline $\begin{array}{l}\text { Stat. } \mathrm{Pa} \text { - } \\
\text { tienten }\end{array}$ & 350 & - & - & (bis 200) & - & (13) \\
\hline $\begin{array}{l}\text { Stat. Pa- } \\
\text { tienten }^{1} \text { ) }\end{array}$ & - & - & - & (bis 185) & - & (14) \\
\hline $\begin{array}{l}\text { Pankreas- } \\
\text { gesunde }\end{array}$ & 39 & - & - & (bis 120) & lognorm & (15) \\
\hline $\begin{array}{l}\text { Pankreas- } \\
\text { gesunde }\end{array}$ & - & - & - & (bis 150) & - & (16) \\
\hline Patienten $^{4}$ ) 1 & 1458 & 59 & (35) & $5-130$ & lognorm & \\
\hline
\end{tabular}

1) Stationäre Patienten ohne Anhaltspunkte für eine Pankreaserkrankung

2) 2-h-Wert, Endpunktstitration, $37{ }^{\circ} \mathrm{C}$ Reaktionstemperatur

3) $\bar{x}+3 s$

$\left.{ }^{4}\right)$ Stationäre und ambulante Patienten ohne Anhaltspunkte für eine Pankreaserkrankung (Scholz und Appel, unveröffentlicht)

Rolitetracyclin (Reverin, $4 \mathrm{~g} /$ ), Aprotinin (Trasylol, $5000 \mathrm{KIE} / \mathrm{ml}$ ); Medikation mit Opiaten und Heparin kann zu einem Anstieg der Lipaseaktivität führen. Störfaktoren sind Eserin, Chinin und Aldehyḍe (3), Atoxyl (4) und gallensaure Salze (4) (vor allem 1. c. (1)).

\section{Biologische Merkmale}

Normbereiche: siehe Tabelle 4.

Alters- und Geschlechtsabhängigkeit: Nicht ersichtlich. Intraindividuelle, tagesrhythmische, jahreszeitliche oder klimatische Schwankungen: weder eigene noch Literaturangaben vorhanden.

Anderes biologisches Material: das Verfahren wurde problemlos angewandt bei Duodenalflüssigkeiten, Bauchpunktatsflüssigkeiten, Wundsekreten und Urin. In mehreren Fällen von Patienten mit schwerer akuter Pankreatitis wurden im Urin methodisch einwandfrei abgesichert geringe katalytische Konzentrationen von Lipase, bis zu $1000 \mathrm{U} / 1$, nachgewiesen. Zur Absicherung dienten Kontrollen mit Validate $\mathrm{N}$, normalen und pathologischen Seren, Verlängerung der Reaktionszeit auf $2 \mathrm{~h}$, Versuche mit erhitztem Urin $\left(10 \mathrm{~min} / 90^{\circ} \mathrm{C}\right)$, vor allem durch Variation der Reaktionsgeschwindigkeit durch Temperaturänderungen im geschlossenen System (Wechsel der Thermostatenflüssigkeit) von 0 bis $50{ }^{\circ} \mathrm{C}$. Auf die widersprüchliche Literatur soll hier nicht eingegangen werden (24). Wundsekrete enthielten bis zu $80000 \mathrm{U} / 1$.

\section{Technische Merkmale}

Praktikabilität: 1 Bestimmung erfordert etwa 20-30 min, eine Serie von 20 Bestimmungen etwa $1-1,5 \mathrm{~h}$ inklusive Kontrollen. Rasche Temperatureinstellung bei etwa 2,5 ml Endvolumen; einfache Handhabung, kein $\mathrm{N}_{2}$-Schutzgas. Gerade diese Abweichung von der Originalmethode wurde sehr sorgfältig durch größ̣ere Vergleichsserien zu begründen versucht, stellt sie doch einen erheblichen Schritt zur Vereinfachung dar (Gasflaschen, Waschflaschen, Natronkalkturm, Blasenzähler entfallen). Es wurden keine statistisch signifikanten Unterschiede der Ergebnisse der Versuchsserien mit und ohne Schutzgas gefunden. Wahrscheinlich ist der Totraum des Reaktionsgefäßes für eine meßbare $\mathrm{CO}_{2}$-Einwirkung zu klein.

Mechanisierungsgrad: eine Teil-oder Vollmechanisierung ist $z$. Z. weder möglich noch vorgesehen.

Andere Methoden: Zur gegenwärtigen Situation bezüglịch Provokations-(Evokations)-Testen siehe 1. c. (5), Chymotrypsin im Stuhl (6); weitere Literatur zur Lipase 1. c. (17-22), im Urin 1. c. (24).

\section{Fehlerkatalog und Funktionshinweise}

Bei der Herstellung des Olivenöls nach Zugabe der $\mathrm{HCl}$ stets homogenisieren; Schütteln oder Rühren allein genügt nicht; sonst zu hohe Leerwerte.

Gummi-arabicum-Lösung gut absitzen lassen, evt. kurz zentrifugieren; sonst Trübungen mit Verstopfungen der Elektroden. Emulsionsreste nach Gebrauch, größere Mengen spätestens nach einer Woche verwerfen; sonst zu hohe Leerwerte.

Titrationskurve mit jeder neuen Charge Gummi-arabicum aufstellen, Bereich pH 4,5-9,0.

Seren mit sehr niedrigem $\mathrm{pH}=$ Wert bei metabolischen Acidosen zuvor auf etwa 8,2 einstellen; sonst Messung außerhälb des pH-Optimums. Vor jeder Messung Lösungen und Seren im Wasserbad auf $25^{\circ} \mathrm{C}$ erwärmen, Temperaturkonstanz kontrollieren; sonst Unlinearität. Das ganze System vor jeder Messung auf luftdichten Verschluß prüfen; sonst zu hohe Werte $\left(\mathrm{CO}_{2}\right)$. Dichtheit des Titrant- 
zuführsystems prüfen, besonders am Drehschalter der Autobürette (Planschliffe!); sonst Vortäuschung hoher Aktivitäten durch Leervorschub der Kolbenbürette und Austropfen des Titranten am Planschliff. Keine Luftblasen im Titrantzuführsystem; sonst zu hohe Werte durch irrtümlichen weiteren Vorschub der Kolbenbürette. Rührmagnet mit den großen Zacken nach oben in das Titriergefäß einlegen; sonst ungleichmäßiges Rühren (Treppenkurven). Nach Auswechseln der Elektroden auf richtiges Einsetzen und richtige Stellung der Elektroden achten; sonst unregelmäßige Titrationskurven durch nicht gleichmäßige Elektrodenreaktionen.

Geometrie der Küvettenanordnung beachten: Küvette muß gut waagerecht sitzen, Elektroden und Zuleitung für Titrant dürfen sich und die Wände nicht berühren; Zuleitung darf nicht zu nahe an den Elektroden sein (Grenzschichtphänomene); sonst ungleichmäßige Titrationskurven.

\section{Literatur}

1. Rick, W. (1969), diese Z. 7, 530-539.

2. Rick, W. (1971), Laboratoriumsdiagnostik der Verdauungsund Resorptionsstörungen, in: Wissenschaftliche Berichte Merck, Band 6, S. 60-130, E. Merck, Darmstadt.

3. Schultis, K. (1974), in: Methodische Fortschritte im medizinischen Laboratorium, Band 2, „Malabsorption und Maldigestion ", (Englhardt, A. \& Lommel, H. ed.), S. 87-114, Verlag Chemie, Weinheim.

4. Englhardt, H. (1974), in: Methodische Fortschritte im medizinischen Laboratorium, Band 2, „Malabsorption und Maldigestion", (Englhardt, A. \& Lommel, H. ed.), S. 115-122, Verlag Chemie, Weinheim.

5. Schmidt, H. \& Witthöft, C. (1976),Leber Magen Darm 6, 227-234, insbes. S. 228.

6. Amman, R. (1976), Leber Magen Darm 6, 217-226, insbes. S. 223.

7. Weber, H. (1965), Disch. Med. Wochenschr. 90, 1170-1174.

8. Schultis, K., Wagner, E. \& Voßköhler, E. (1973), Dtsch. Med. Wochenschr. 98, 364.

9. Voßköhler, E. (1971), Dissertation, Gießen

10. Goebell, H. (1970), Internist 11,117.

11. Goebell, H., Bode, Ch. \& Lemberg, G. (1969), Dtsch. Med. Wochenschr. 94, 2086.

12. Wagner, E., Irfanoglu, E. M. \& Schultis, K. (1971), Chirurg $42,162$.

13. Olbermann, M., Prellwitz, W. \& Recke, S. (1973), Dtsch. Med. Wochenschr. 98, 8 .
Titrationsgefäße, Rührer und Elektroden mit Wasser, Aceton und Benzin reinigen. Küvette, Rührer und Elektroden nach dem Reinigen gut trocknen lassen. GlasPlanschliff alle Wochen reinigen und neu fetten. Tintenschreiber alle vier Wochen reinigen.

Elektroden in Wasser eingetaucht verwahren. Kalomelelektrode von Zeit zu Zeit mit feinstem Schmirgelpapier aufrauhen, mit $\mathrm{KCl}$-Lösung nachfüllen. Glasund Kalomelelektrode alle zwei Wochen über Nacht in Pepsin-Salzsäurelösung $(0,1 \mathrm{~mol} / 1 \mathrm{HCl})$. Neue Kalomelelektroden: vor allem bei Auskristallisationen auf Luftfreiheit achten; sonst Titration durch äußeren Feldeinfluß unmöglich.

Glaselektrode: evt. Luftblasen nach oben schütteln; sonst treppenförmige Kurven.

Auf Kondenswasser im Titrant-Vorratsgefäß achten; sonst zu hohe Werte.
14. Stahlheber, H. \& Forell, M. M. (1971), Mitt. Dtsch. Ges. Klin. Chem., Heft 1, S. 4.

15. Bornstein, W., Gebhardt, E. \& Mann, W. (1976), Med. Klin. 71,288

16. Merck, E., Klinisches Labor (1974), S. 201, 12. Aufl. E. Merck, Darmstadt.

17. Bergmeyer, H. U. (1970), Methoden der enzymatischen Analyse, S. 781, 2. Aufl. Verlag Chemie, Weinheim.

18. Curtius, Ch. \& Roth, M. (1974), Clinical Biochemistry, Band 2, S. 1253, Verlag W. de Gruyter, Berlin.

19. Ramiz, A., Nipper, H. C., Merchant, C. R. \& Knoblock, E. C. (1974), diese Z. 12, 285.

20. Hillmann, G. \& Weidemann, G. (1973), diese Z. 11, 257.

21. Berndt, W., Glashoff-Andresen, M., Kapaun, W. \& MüllerWieland, K. (1975), 86. Tagung, Nordwestdeutsche Ges. Inn. Med.

22. Bornschein, W., Gebhardt, E. \& Mann, W. (1976), Med. Klinik, 71, 288-291.

23. Sémériva, M. \& Desnuelle, P., in: Horizons in Biochemistry and Biophysics, (Quagliariello, E., Palmieri, F. \& Singer, Th. P. ed.), Vol. 2, S. 32-59, Addison-Wesley Publ. Comp., London, Amsterdam u. a. (1976).

24. Gülzow, M., Leithäuser, W. \& Trettin, H.-J. (1967), Dtsch. Z. Verd. Stoff wechselkrankh. 27, 109-115.

Priv.-Doz. Dr. W. Appel Südendstraße 32 D-7500 Karlsruhe 
